\title{
EVADE! REFLEXIONES EN TORNO A LA POTENCIA DE UN ESCRITO
}

\author{
Evade! Reflections on the power of a writing
}

\section{RESUMEN}

El propósito de este texto es mostrar la relevancia socio-política del escrito con el que se dio inicio al estallido social chileno de octubre 2019: "Evade!". Utilizando las herramientas de la antropología de la escritura analizo los principales rasgos textuales de este escrito breve, junto con sus condiciones de aparición y de propagación en la ciudad, para así caracterizar su potencia pragmática. El análisis permite comprender la profunda adecuación de ese escrito a las modalidades de funcionamiento del neoliberalismo chileno contemporáneo, pero también su capacidad de sugerir pistas para entender las condiciones de emergencia de una nueva sensibilidad política.

Palabras claves: escrituras expuestas; estallido social; neoliberalismo; performatividad; política.

\section{UNIVERSUM}

Revista de Humanidades y Ciencias Sociales

\section{LUIS CAMPOS MEDINA}

Sociólogo (Universidad de Chile), Máster en Ciencias Sociales (EHESS) y Doctor en Sociología (EHESS). Académico del Instituto de la Vivienda de la Universidad de Chile. Investigador Adjunto Núcleo Milenio Arte, Performatividad y Activismo, Instituto de la Vivienda de la Universidad de Chile.

correo electrónico:

luiscampos@uchilefau.cl

ORCID: 0000-0002-5157-4974

ResearchGate: Luis Campos Medina Scholar.google:

Academia.edu: LuisCamposMedina

Este trabajo forma parte del Núcleo Milenio Arte, Performatividad y Activismo, financiado por la Iniciativa Científica Milenio (ICM), del Gobierno de Chile.

Artículo recibido el 31 de marzo, 2020. Aceptado el 25 de mayo, 2020.

DOI:

Web: http://universum.utalca.cl | ISSN: 0716-498X - 0718-2376 


\begin{abstract}
The aim of this text is to show the socio-political relevance of the writing with which the Chilean social outbreak of October 2019 began: "Evade!". Using the tools of the anthropology of writing, I analyze the main textual features of this brief writing, as well as its conditions of appearance and propagation in the city, in order to characterize its pragmatic potential. The analysis allows us to understand the profound adequacy of this writing to the operating modalities of contemporary Chilean neoliberalism, but also its capacity to suggest clues to understand the conditions for emergence of a new political sensibility.
\end{abstract}

Keywords: exposed writings; social outbreak; neoliberalism; performativity; politics.

\title{
PRESENTACIÓN
}

El origen del llamado "estallido social" de octubre 2019, que sigue afectando a Chile, a pesar de la crisis global gatillada por el Coronavirus, ha sido fechado en un viernes 18 de octubre. Ese día, la ocupación de un conjunto de estaciones del metro de Santiago hizo colapsar el sistema de transporte de la ciudad, convirtiendo la que parecía una tranquila tarde de primavera en un enorme desorden vial y en un singular desbarajuste para muchos de sus habitantes.

Esa tarde de viernes, sin embargo, tuvo antecedentes. Su antesala puede encontrarse en el actuar de cientos de estudiantes secundarios que, varios días antes y frente al alza de la tarifa, comenzaron a evadir el pago del boleto de acceso a la red de metro y conminaron a otros a hacer lo mismo. El llamado era a evadir. Estaciones de metro, paraderos de buses y muros de calles comenzaron a ver la proliferación de un texto. Uno sencillo, simple y directo: "EVADE!".

En este breve ensayo quisiera mostrar que el texto "EVADE!", a pesar de su sencillez, y especialmente debido a su proliferación en la ciudad 
de Santiago, tanto en la antesala al 18 de octubre, como en los días que le siguieron, posee una potencia pragmática inusitada. Con base en esos elementos quisiera proponer que constituye una valiosa pieza gráfica para intentar una comprensión sociológica de los elementos que dieron forma a la explosión social de octubre.

\section{PRELUDIO CONCEPTUAL: HERRAMIENTAS PARA EL ANÁLISIS DE ESCRITURAS EXPUESTAS URBANAS}

Los fundamentos teóricos de mi investigación se encuentran en la perspectiva desarrollada a partir del trabajo pionero del historiador italiano Armando Petrucci (1993). Petrucci fue uno de los primeros en prestar atención a la relevancia e incidencia de las escrituras expuestas en la antigua Roma, mostrando cómo en esas situaciones la escritura asume funciones de exteriorización y de solemnidad.

Ese trabajo ha sido continuado y prolongado por la antropóloga Béatrice Fraenkel, quien ha sistematizado un enfoque para dar cuenta de los usos y los efectos que generan los escritos expuestos en los espacios públicos de una sociedad determinada. La antropología de la escritura busca superar las aproximaciones tradicionales a los escritos, que tienden a concentrarse en el análisis de la significación y la interpretación, para preocuparse por el rol que juegan en la ecología de las situaciones y sobre las características y condiciones de su agencia (Denis y Pointille, 2013). Por ejemplo, las informaciones escritas en una estación de trenes, un terminal de buses o un aeropuerto, sea en carteles hechos a mano o en pantallas de alta tecnología, contribuyen a dar forma a nuestra experiencia en estos lugares: nos permiten planificar nuestras acciones, determinar el ritmo de nuestros desplazamientos y el tipo de relación que establecemos con nuestros acompañantes. A veces, incluso, nos obligan a hacer cosas, como cambiarnos de andén o ir a tomar contacto con los funcionarios de una empresa de transportes.

Fraenkel (2002/2009/2013) habla de "escrituras expuestas", englobando en esa categoría una serie de escritos en el espacio público que 
han sido efectuados en una superficie visible por otros (por eso el adjetivo expuestas) y prestando especial atención a su condición material (la escritura supone una inscripción y un trabajo; el escrito deviene un artefacto). Desde esta perspectiva, las escrituras expuestas urbanas tienen un rol discreto pero fundamental: todas ellas aspiran a ser observadas o leídas por alguien y buscan generar repercusiones sobre sus observadores o lectores. No son inocuas, sino que intentan activar procesos, desencadenar acciones. En el plano de la acción política, Araya (2007/2008/2010) describe cómo el escrito permite condensar sentidos y canalizar una acción contestaría a un régimen dictatorial, interpelando a las y los transeúntes que lo encuentran en su camino. En un registro diferente, hemos mostrado cómo estos escritos, en situaciones de desastres y catástrofes, buscan interpelar a sus lectores para solicitar ayuda o coordinar la acción de reconstrucción (Campos 2014; Campos y Suazo 2019).

Como propone Fraenkel, las inscripciones gráficas expuestas hacen del espacio público un "espacio de influencia" (Fraenkel, 2002). Más aun, estas inscripciones sugieren que cualquier acción efectuada en el espacio público, por ínfima que parezca, como puede ser un altar hecho de cientos o miles de post-it luego de la destrucción de las torres gemelas en Nueva York, en 2001 (Fraenkel, 2002), involucra una concepción sobre los métodos a través de los cuales este es producido, apropiado y controlado (Artières, 2013). Supone, también, la realización de una serie de operaciones que en muchos casos resultan invisibles: un verdadero trabajo en torno al escrito (Latour y Hermant,1998).

Los análisis de escrituras expuestas incluyen un "conjunto de inscripciones tales como los slogans políticos, los tags, los graffitis obscenos o los de amor, los afiches publicitarios que adornan nuestras ciudades y coexisten con las producciones gráficas más solemnes" (Fraenkel, 2009: 158). Las escrituras expuestas ponen de manifiesto temas y contenidos relevantes para un determinado grupo, pero también atestiguan de las normas gráficas que orientan la producción de los textos, así como de "las relaciones de dominación de ciertos grupos sociales sobre otros" (Fraenkel, 2009: 158).

De acuerdo con la perspectiva de aquí esbozada, las escrituras expuestas 
pueden ser observadas a través de tres nociones principales: visibilidad, legibilidad y publicidad (Fraenkel, 2002/2007/2009). La primera de ellas, la visibilidad, indica que este tipo de escrituras se ofrece a la mirada de todos y que, considerando que en las ciudades hay múltiples signos que compiten por la atención de los transeúntes, captar esa atención requiere seleccionar emplazamientos adecuados y construir objetos atractivos (Fraenkel, 2002). La segunda, la legibilidad, apunta a que los mensajes deben adecuarse a normas gráficas y textuales, lo que se traduce en que los escritos urbanos usualmente sean breves, repetitivos y simples, mostrando en toda su potencia el arte de la abreviación (Fraenkel, 2002). Además, suele ocurrir que los escritos urbanos son leídos entre varios y a distancia (Fraenkel, 2009). La tercera, la publicidad, plantea que dichas escrituras se presentan en diversos lugares del espacio urbano, convirtiéndolo en espacio de lectura y escritura. En la ciudad, dado este carácter público, el encuentro con escrituras expuestas es en buena medida imprevisible (Fraenkel, 2002).

Acompañando a estas tres características, la autora destaca un cuarto rasgo: las escrituras expuestas buscan hacer cosas sobre quienes las leen, por lo que en su análisis vale tener en cuenta su performatividad. Sabemos que cualquier forma de escritura está siempre dirigida a otros. No obstante, este carácter se ve intensificado en el caso de las escrituras expuestas, ya que, aún cuando no se trata de un destinatario específico, ni claramente establecido, como en el caso de una carta u otro envío postal, se aspira a la lectura de todos aquellos que se encuentren en el radio de influencia del escrito (Campos,2009/2014/2019). Ese radio de acción depende, por cierto, del tamaño de la escritura y del tipo de letra, pero también y especialmente, del emplazamiento de la escritura, por cuanto "la opción de un lugar de exposición puede conferir al escrito mismo una fuerza suplementaria" (Fraenkel, 2009: 159). Las escrituras expuestas, muchas de ellas generadas de forma ilegal y con una duración efímera, son producidas para "informar, para conmemorar, para honrar. Son también producidas para actuar sobre sí mismo, para reconfortarse, convencerse o hacer el duelo" (Fraenkel, 2002: 23). ${ }^{1}$

1 Traducción del autor. 
Para dar cuenta del carácter pragmático de las escrituras expuestas, el punto de partida se encuentra en la conceptualización de Austin (2003; Alarcón, 2008) y su teoría de los actos de habla, continuada y desarrollada por Searle (1982/2001). Solo que, reparando en un aspecto olvidado por ambos filósofos, e incluso por sus críticos (Benveniste, 1976): el escrito supone una materialidad, un emplazamiento, una producción. Se trata de comprender cómo los escritos hacen cosas, incluso algunas imprevistas, tal como sugiere Austin con su noción de acto perlocucionario, de acuerdo a la cual un acto de lenguaje puede producir "consecuencias o efectos sobre los sentimientos, pensamientos o acciones del auditorio, o de quien emite la expresión, o de otras personas" (Austin, 2003: 145). Solo que, para entender esos efectos, no basta con efectuar un análisis textual de los escritos, sino que es necesario interrogarse, entre otras cosas, acerca de los modos de aparición del escrito (las antes mencionadas visibilidad, legibilidad y publicidad), su temporalidad y permanencia, su capacidad de integrarse a paisajes habituales de transeúntes o habitantes, su habilidad para interpelar a sus destinatarios. En síntesis, su capacidad para actuar sobre otros y desencadenar efectos. Esta concepción del escrito resuena con discusiones conceptuales producidas en otros terrenos, donde se analiza la agencia de entidades no humanas, como los análisis de cultura material, entre los cuales cabe mencionar el ejemplo de Pellegram (1998), quien muestra cómo la materialidad del papel es un elemento crucial a tener en consideración en el estudio de los escritos de la esfera del trabajo, pues ella está ligada a significados, a modalidades de uso y a jerarquías laborales. La relevancia de la materialidad es justamente el foco de la obra del mismo nombre, "Materiality", publicada por Daniel Miller en la primera década del milenio (2005) y un aspecto que cruza la edición de una de sus obras anteriores (Miller, 1998). En ambos, el autor destaca la capacidad de actuar sobre nosotros que poseen los objetos de nuestro entorno, incluso en función de rasgos — como la materialidad — que a veces damos por descontados. 


\section{DEL INTERÉS DE UNA PALABRA}

Situados en estas coordenadas conceptuales, podemos, ahora, sumergirnos en el análisis del texto considerado: "EVADE!", vale decir, el verbo evadir, en la segunda persona singular del modo imperativo, acompañado de un signo de cierre de exclamación (“!”).

Se vuelve necesario hacer aquí una aclaración. En mi trabajo de terreno, que consistió en el recorrido regular y el registro fotográfico sistemático de los alrededores de la Plaza de la Dignidad (ex Plaza Baquedano, más frecuentemente denominada Plaza Italia) durante los dos meses siguientes al 18 de octubre, así como de otros sectores de las comunas de Ñuñoa, Macul y Estación Central, encontré el escrito "evade" en variadas versiones, siendo la más frecuente aquella en que estaba escrito en letras capitales (mayúsculas) y que incluía un signo de exclamación al final: "EVADE!", aunque también era habitual encontrarlo sin el cierre de exclamación o con varios de ellos (!!!). Vi ratificada esta constatación al revisar registros fotográficos de otros colegas. Consagro este análisis a la versión que incluye el signo de exclamación, aunque el razonamiento también aplica a las versiones en que este último no aparece.

Decíamos anteriormente que las escrituras expuestas en el ámbito urbano suelen caracterizarse por su brevedad. Fraenkel indica que muchas veces asumen un estilo "lapidario", recurren a las "fórmulas" o adoptan el carácter de eslóganes (Fraenkel, 2002). En el caso de "Evade!", estamos frente a un texto muy breve, compuesto solo de una palabra y un signo de exclamación al final. Efectivamente, esa brevedad hace pensar en su funcionamiento como eslogan, es decir, como una fórmula sintética y original, como las que utiliza la publicidad o la propaganda política, cuyo propósito es hacer resonar un mensaje entre una audiencia. Su modalidad imperativa hace recordar a la señalética, que es el principal tipo de escritura que utiliza el modo imperativo en la urbe. El signo de cierre de exclamación viene a reforzar ese carácter de mandato. 
Fotografía n⿳1

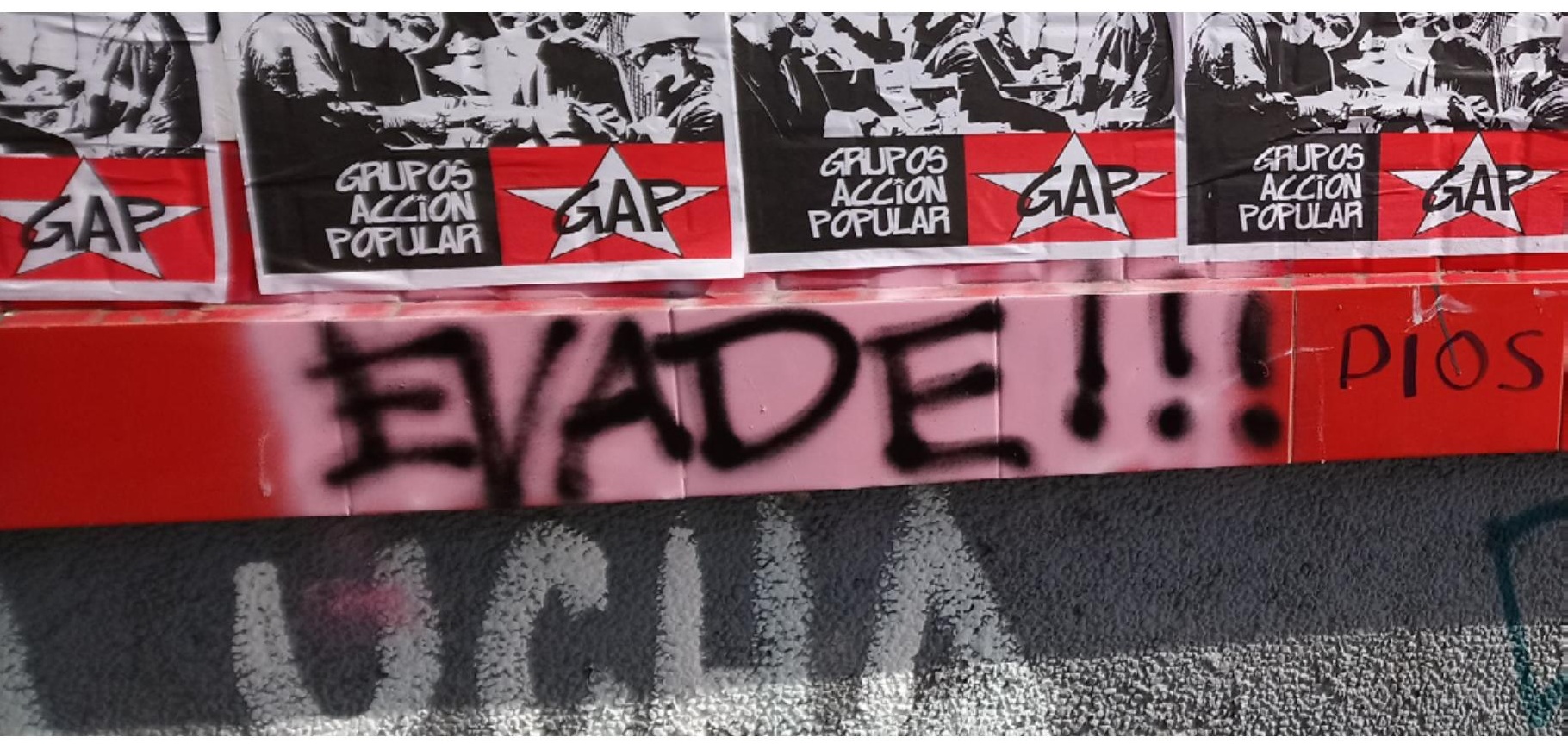

Fuente: fotografía del autor

Brevedad, modalidad imperativa y exclamación. He aquí los principales rasgos textuales de este escrito. Si recurrimos a la tipología de actos de habla desarrollada por Searle (1982) podemos añadir que se trata de un "directivo", vale decir, de un texto que se caracteriza por: a) constituir "un intento por parte del hablante para que el oyente haga algo"; b) que busca que "el mundo se ajuste a las palabras" y; c) que tiene, como condición de sinceridad, el "querer" o “desear” (Searle, 1982: 53). Este carácter de directivo es fundamental, puesto que indica que estamos frente a un texto cuya relevancia no se juega en su verdad o falsedad respecto de lo que ocurre en el mundo, sino que busca hacer que el mundo se adecúe a las palabras, es decir, busca producir un ajuste de la realidad. ¿De qué ajuste estamos hablando?

Un primer aspecto relevante se encuentra en el uso de la segunda persona del singular, pues hay allí una definición implícita del destinatario del enunciado: no se trata de un colectivo o un grupo, sino que de un individuo. No hay indicación de género, lo que refuerza el carácter indiferenciado del 
destinatario: cualquiera que lea el mensaje se convierte en su objetivo y es a él o ella a quien se compele a hacer algo, en este caso, evadir.

De acuerdo al diccionario de la Real Academia Española (RAE), el verbo evadir posee cinco definiciones. La primera lo entiende como evitar un daño o peligro. La segunda, como eludir con arte o astucia una dificultad prevista. La tercera, como sacar ilegalmente de un país dinero o cualquier tipo de bienes. La cuarta, como fugarse o escaparse. Y la quinta, como desentenderse de cualquier preocupación o inquietud. Atendiendo a lo dicho anteriormente respecto de las características del enfoque de la antropología de la escritura, para determinar si alguna de estas definiciones remite al escrito aludido no basta con ensayar una interpretación textual, sino que debemos referirnos a sus condiciones de aparición en la ciudad. A esto dedicaremos la siguiente sección.

\section{CONDICIONES DE APARICIÓN DE UN ESCRITO EN LA URBE}

Como indicamos concisamente al inicio de este texto, días antes del 18 de octubre varias estaciones de metro, paraderos de buses y muros de calles comenzaron a ver la proliferación del escrito “EVADE!". La secuencia propuesta en la frase anterior busca sugerir la sucesión real de aparición del escrito en el espacio urbano, comenzando por las estaciones de la red de metro, siguiendo por los paraderos del transporte de superficie y continuando por los muros de las calles de la ciudad. Indicamos, además, que los actores movilizados en los días previos al 18 de octubre eran las y los estudiantes secundarios y que el foco de su movilización era el alza de la tarifa del pasaje de metro.

Este conjunto de antecedentes opera como el trasfondo interpretativo del mensaje (Searle, 1997) y permite precisar su significado. No obstante, un elemento crucial en esa especificación de sentido radica en el emplazamiento inicial del escrito: las estaciones de metro. Se trata de evadir el pago del boleto de metro. En esa dirección, la evasión puesta en juego se relaciona con las definiciones segunda y cuarta del verbo evadir, aunque no se circunscribe 
completamente a ninguna de ellas (eludir con arte o astucia una dificultad prevista y fugarse o escaparse, respectivamente).

Pero el emplazamiento de un escrito expuesto es un aspecto aún más relevante. En este punto Fraenkel (2007) sigue a Bosredon (1997) y su teoría del etiquetaje para mostrarnos que la ubicación de un escrito muchas veces le otorga parte de su significado, su fuerza enunciativa, hasta llegar, en ocasiones, a establecerse una relación de coexistencia y co-determinación entre texto y emplazamiento. Más aún, como señalan Denis y Pointille (2013), en ocasiones se produce una articulación constitutiva entre escrito y espacio,

como en el caso de los paneles de dirección, los letreros de calles, ciertos afiches de seguridad (...) [cuya] significación está intrínsecamente ligada a su emplazamiento, así como el lugar que habitan está en gran medida definido por la presencia del escrito (Denis y Pointille 2013: 21). ${ }^{2}$

Este punto, que puede parecer trivial, encierra importantes consecuencias epistemológicas, ya que, tal como indican los autores comentados (Denis y Pointille, 2013), esto supone “abandonar una definición académica del escrito", que tiende a concentrarse únicamente en la textualidad, para pasar a preguntarse, como dijimos anteriormente, sobre el rol que juegan los escritos en "la ecología de las situaciones" y sobre su particular forma de agencia.

Entender el efecto pragmático del emplazamiento inicial del escrito "EVADE!" en las estaciones del metro, y de su posterior difusión en la ciudad, requiere de una indicación acerca de las características de la “atmósfera gráfica” (Fraenkel, 2002) de esos espacios. En otro lugar hemos dado cuenta detallada de la peculiar atmósfera gráfica de la red del Metro de Santiago (Campos y Aguilar, 2015). De manera resumida, podemos indicar que dos de sus rasgos principales se encuentran, primero, en el predominio y casi exclusividad de dos tipos de escritos: publicidad y señalética y, segundo, en un acérrimo control del surgimiento y proliferación de otro tipo de escritos, particularmente los ilícitos, a través de prácticas sistemáticas de borrado y limpieza.

2 Traducción del autor. 
La aparición y proliferación del escrito "EVADE!" constituye, en consecuencia, una intervención de esa atmósfera gráfica, ya que rompe con el orden y la regularidad que la caracterizan, pero además sugiere que hay productores de escritos que contravienen las normas gráficas de los detentadores del poder en la red de metro y que incitan a un comportamiento que, a su vez, contraviene las normativas de control de los cuerpos en la red.

En efecto, el hecho de no poder observar huellas o registros de usos humanos en la red de metro, menos aún de rayados, denota una restricción del espectro de interacción corporal con el espacio de las estaciones y vagones, así como una reducción de las formas potenciales de intervención de ese espacio.

Considerando que los modos de uso de los cuerpos también están fuertemente regulados, el escrito "EVADE!" constituye una doble irritación: alguien ya violó la norma para hacer el rayado y está incitando a otros a que violen la regla del pago. Hay aquí un interesante efecto de simetría entre la intervención de las normas de control gráfico y las normas de control "somático" (Shusterman 2015).

Siguiendo este mismo razonamiento, cabe plantear que la difusión del escrito hacia los paraderos de la red de buses y hacia los muros de las calles de la ciudad implica una ampliación del sentido de la evasión. Se puede pensar ahora en evadir el pago en la red de transporte de superficie, pero se puede pensar también en una forma de evasión que ya no tiene una referencia directa determinada por el emplazamiento del escrito. En este sentido, la proliferación del escrito a través de muros indiferenciados sugiere, a su vez, una expansión y generalización de la incitación a evadir y una ampliación de su espectro de resonancia. En este punto es fundamental el hecho de que el escrito "EVADE!" comienza a convivir con una multiplicidad de otros escritos, que le dan forma pública a las motivaciones y justificaciones de la movilización, de la contestación y del desacato (Denis y Pointille, 2009; Latour y Weibel, 2005). Esa variedad y diversidad de escritos comienzan a adquirir su significado, no solo de su contenidoproposicional, sinoque también desu co-existencia enunmismomuro. 


\section{Fotografía $\mathbf{n}^{0} 2$}

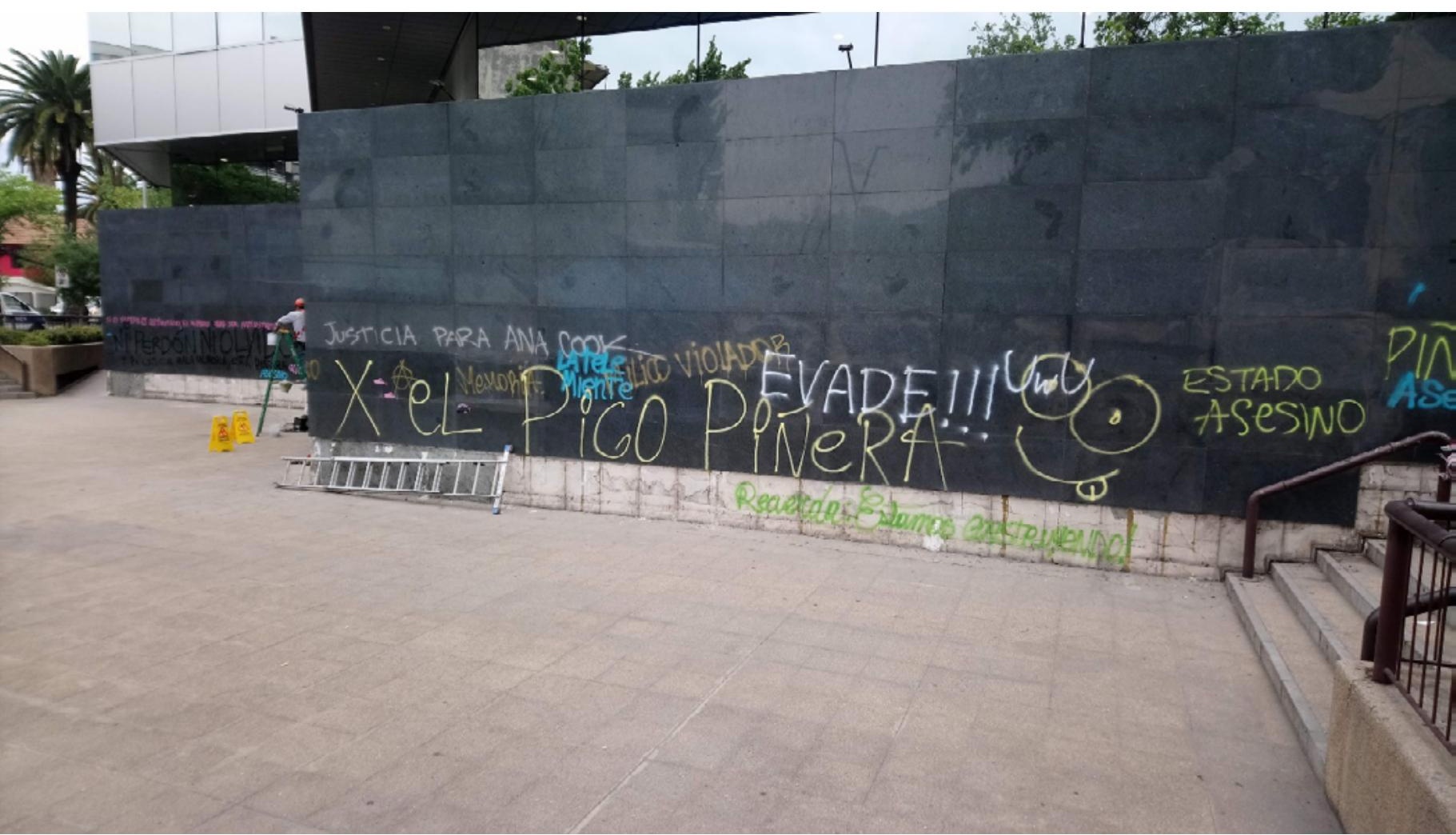

Fuente: fotografía del autor 


\section{Fotografía n⿳0}

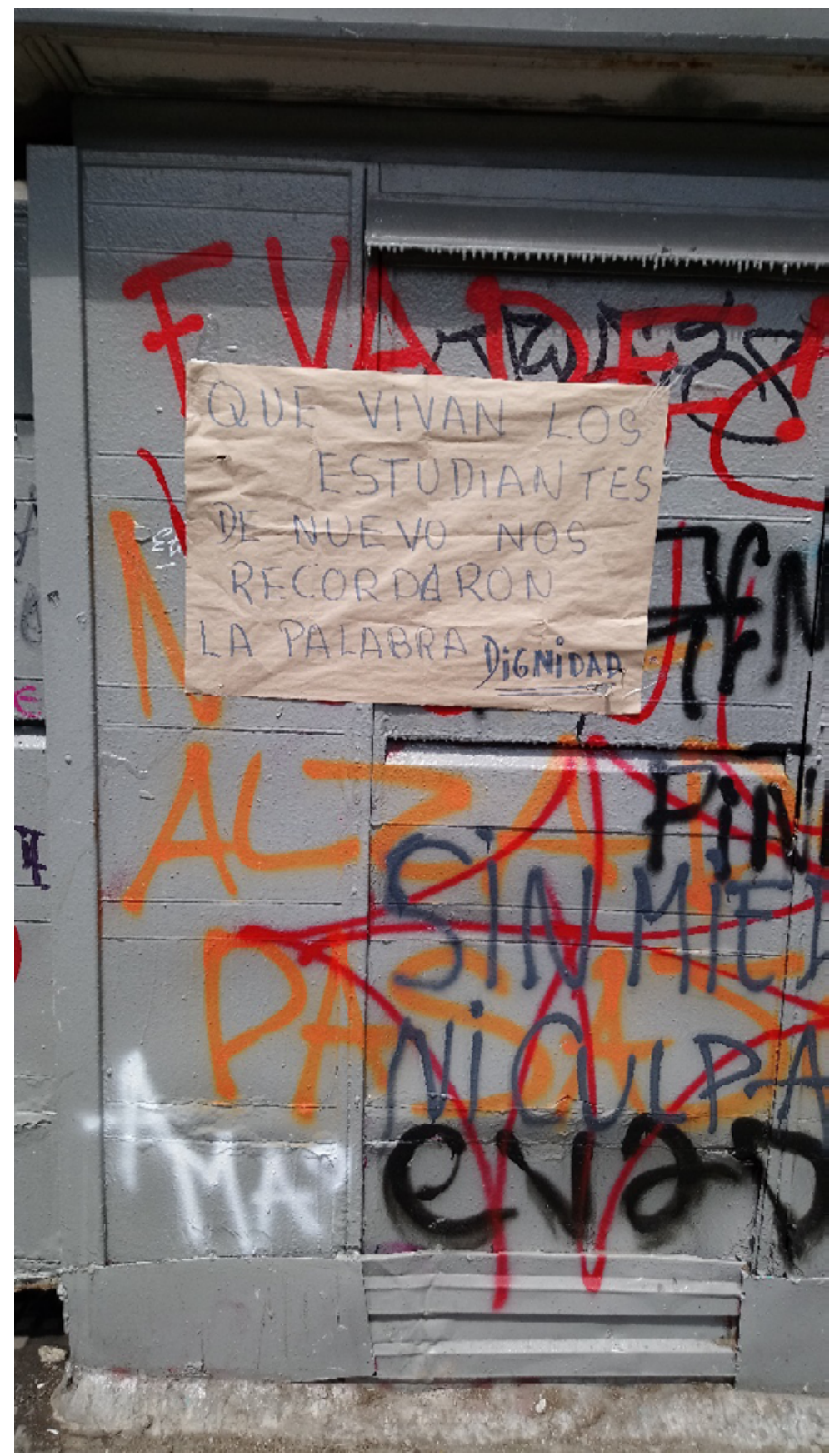

Fuente: fotografía del autor 
Conectando el rasgo imperativo y conminatorio del texto con las características del proceso de aparición y proliferación del escrito en la ciudad, resulta pertinente proponer la idea de una verdadera "propagación gráfica", en la medida que se trata de una transmisión por proximidad, de lector en lector, que actúa como la difusión de un rumor o de una información, pero también, por cierto, que busca generar efectos en el lector, persuadirlo, hacerlo actuar. Jugando un poco con el concepto, podemos pensar que la propagación implica una desconexión del "enclave pragmático" (Schaeffer, 1999/2009/2013) inicial del escrito (la red de metro) y, en consecuencia, una expansión de su disponibilidad de uso, que se potencia en su articulación con otros escritos expuestos (los otros escritos que llaman a la protesta, la contestación y el desacato). Un rasgo que a su vez refuerza esta potencia pragmática se encuentra en la inexistencia de un autor específico para el escrito "EVADE!". Es lo que veremos a continuación.

\section{AUTORÍA Y POLIGRAFÍA}

Efectivamente, otra característica importante de observar en la propagación gráfica del escrito "EVADE!" se encuentra en que no posee un autor: no hay un nombre ni una firma asociada, como tampoco hay un color específico que se reitere u otra señal que insinúe que tras de él hay un autor específico. Más aún, el uso de las mayúsculas juega aquí un rol importante pues cumple la función de atenuar las particularidades del manuscrito, ofreciendo una forma estandarizada de escritura.

Las formas de aparición del escrito también se vuelven importantes aquí, puesto que no se trata de escritos monumentales, sino que mayoritariamente de escritos de escala individual, esto es, que parecen elaborados por una persona y no por un grupo o un equipo, como en el caso de murales o grafitis más sofisticados. El sujeto de la enunciación, por recuperar los términos de la teoría de los actos de habla, es presumiblemente una persona, un individuo, pero, como acabamos de indicar, no disponemos de señales que nos permitan identificarlo. Tampoco sabemos si tras de cada escrito se encuentra el mismo 
autor. Más bien ocurre algo distinto: cada "EVADE!" puede haber sido escrito por un individuo distinto, que puede ser cualquiera pero que, a pesar de ser diferentes, han decidido escribir lo mismo.

En este sentido, las condiciones de aparición del escrito - su materialidad, visibilidad, legibilidad, publicidad - vuelven plausible aplicar la hipótesis de la "poligrafía" (Fraenkel, 2002) para dar cuenta de que se trata de una enunciación entre varias personas, no necesariamente coordinadas, lo que la vuelve múltiple y potencialmente multiplicable. Dicho de otra forma, la constatación de los rasgos de des-singularización del escrito y de incremento en el potencial imitativo del mismo, hacen pertinente pensar en una pluralidad de autores. La relevancia pragmática de este carácter poligráfico se encuentra en que cualquier lector se convierte en un potencial autor del escrito, lo que también puede ser enunciado como la capacidad de generar disposiciones para la acción en todos quienes se constituyen, situacionalmente, en sus receptores.

Esto permite posicionar la idea del texto como un artefacto sensible que entrecruza lenguaje y acción, y que, a través de sus incitaciones es capaz de activar resonancias, contribuyendo a la puesta en cuestión del orden social y a la re-construcción colectiva de valores y creencias en ese orden social. Al abordaje de esas resonancias consagraremos el siguiente apartado.

\section{RESONANCIAS SOCIO-POLÍTICAS DE UN ESCRITO}

Retomando y complementando el análisis planteado anteriormente en la sección consagrada al análisis textual del escrito "EVADE!", un primer elemento en el que es importante detenerse es en el verbo empleado: evadir. En efecto, resulta por lo menos curioso utilizar un verbo que no remite a una forma de contestación, de oposición o de conflicto directo respecto del sistema, como podría ser combatir, derribar, destruir, luchar o resistir, sino que más bien da cuenta de una forma paradójica de relación con el mismo (paradójica considerando las escrituras políticas más habituales). Sin embargo, la selección de ese verbo resulta especialmente significativa a la luz de los análisis sobre el neoliberalismo, en especial los contemporáneos. 
A fines de la década de los setenta Michel Foucault dicta su curso sobre el nacimiento de la biopolítica en el Collège de France (2004). Ese curso constituye un hito en el análisis del neoliberalismo y parte de su influencia sobre los trabajos posteriores se encuentra, justamente, en proporcionar los elementos para pensar el neoliberalismo como una verdadera política orientada a la vitalidad de la especie humana. No obstante, esta perspectiva no fue cabalmente desarrollada por Foucault.

Este vacío es, en buena medida, el punto de partida para el trabajo de Barbara Stiegler (2019), titulado Il faut s'adapter, cuya traducción al español correspondería, más o menos, la conminación "Hay que adaptarse". Adoptando una perspectiva genealógica, la obra de Stiegler busca mostrar que uno de los rasgos fundamentales del neoliberalismo, a lo largo de su historia de más de ochenta años, pero que se acentúa en las últimas décadas, es el imperativo a la adaptación. Stiegler muestra cómo ese imperativo se entronca en concepciones derivadas de la teoría de la evolución de Darwin $y$ constituye un aspecto crucial en el pensamiento neoliberal que no fue abordado por Foucault: la alta complejidad del entorno social, económico y cultural generado por el desarrollo y expansión del capitalismo instala un desafío nunca antes visto para la especie humana y supone la generación de habilidades y destrezas que superan las capacidades adaptativas naturales o espontáneas que hasta ahora han mostrado los seres humanos. Por ello se requiere de una acción intencionada y dirigida a generar dicha adaptación y no una promoción de libertades, como hace el liberalismo. Por el contrario, se necesita un "retorno invasivo de la acción del estado en todas las esferas de la vida social" (Stiegler 2019: 12). ${ }^{3}$ En consecuencia, desde este punto de vista, el imperativo a la adaptación no es una cuestión accesoria al neoliberalismo, sino que absolutamente medular y el rol del estado en ello, a pesar de pretender pasar desapercibido, es clave.

Si el imperativo adaptativo es un aspecto crucial del neoliberalismo, el llamado a la evasión efectuado por el escrito "EVADE!" adopta otro cariz: se

3 Traducción del autor. 
trata de una conminación a actuar de manera opuesta a lo que el neoliberalismo propone, no adaptarse a las condiciones del entorno socio-material, sino que evitarlas y eludirlas. Aquí vuelven a ser pertinentes las definiciones del verbo evadir indicadas anteriormente, en tanto "EVADE!" podría estar insinuando que aquello que se debe evadir no es solo el pago de un boleto de metro, sino que un sistema que provoca dificultades (segunda definición de la RAE), o algo que incluso puede provocar daño o peligro (primera definición de la RAE). Más aún, tal vez lo que se busca evadir es un sistema que busca regular los siempre "defectuosos" deseos de la especie humana, tal como pretende el neoliberalismo, en la lectura de Stiegler (2019: 227).

Más aún, la autora nos muestra cómo este imperativo adaptativo está usualmente asociado a una retórica del retraso permanente en las distintas esferas de la vida social, así como a la necesidad de una aceleración de los comportamientos y las reacciones individuales dirigida a resolver ese retraso, pero también a convertirse en el medio para alcanzar la realización personal. Se trata de la articulación de dos puntos fundamentales del neoliberalismo, a nivel del sistema y a nivel del individuo, respecto de los cuales el llamado a evadir resuena como una contestación. Desde esta interpretación, no habría que ajustarse o apurarse para que el sistema funcione, no habría que intensificar nuestras acciones o empeños para conseguir aquello que nos hace felices. Más bien habría que salir del engranaje del sistema y desactivar nuestras conminaciones interiores a conseguir los satisfactores promovidos por este.

Un segundo elemento textual relevante de atender es el uso de la segunda persona singular, puesto que, como señalamos anteriormente, constituye un direccionamiento individual del destinatario de la conminación. Esto implica que no se busca movilizar ni a un grupo organizado, ni al sujeto histórico de otras épocas, sino que, a cualquier individuo, a pesar de que todos ellos sean el resultado de la acción del neoliberalismo.

La sociedad chilena actual está plagada de conminaciones al actuar individual en los planos del trabajo, la salud, el acceso a la vivienda o al crédito bancario, por mencionar solo algunos. Más aún, una premisa del funcionamiento del sistema es el actuar de un individuo responsable de sí mismo, puesto que, 
como indica Garretón, en el neoliberalismo "el individuo es comprendido como el principal responsable de sus actos" (2012: 24-25) o, como subraya Araujo, el neoliberalismo "introdujo la imagen de una sociedad perfectamente móvil y competitiva; la valoración de la ambición personal y la confianza en el esfuerzo propio; la imagen de actores fuertemente responsabilizados de su destino personal" (2019: 18-19). Hay aquí, en "EVADE!", una suerte de tergiversación paradójica del imperativo a la adaptación: se mantiene la premisa del actuar individual, pero ya no para responsabilizarse, sino que para desentenderse, para eludir la andanada de conminaciones morales que inundan la vida cotidiana de los sujetos neoliberales chilenos: esfuerzo personal, sujeto de crédito, responsable de sus actos o capacidad de aguante. No parece exagerado plantear aquí una suerte de subversión contra el disciplinamiento de las individualidades. Una manera de desactivar la potencia de los mandatos que nos mueven y que suelen comenzar con un "hay que hacer" esto o lo otro.

El uso de la segunda persona singular pareciera estar basado en un diagnóstico sociológico respecto de la individualización de las trayectorias y la descomposición de los colectivos, pero, al mismo tiempo, es un escrito cuya efectividad depende del funcionamiento de un trasfondo interpretativo (Searle, 1997) que reposa en un entorno compartido: las condiciones que ha generado el propio neoliberalismo y que son, a la vez, materiales y simbólicas, constrictivas y habilitantes. Dicho en el lenguaje de Austin (2003), parte de las condiciones de felicidad del acto de lenguaje dependen del propio actuar del neoliberalismo en las individualidades que ha exacerbado la tendencia a pensarnos de modo individual y aislado.

Untercerrasgotextual relevanteseencuentraenelusodelmodoimperativo y la exclamación. Profundizando lo recién planteado, el diagnóstico sociológico subyacente parece incluir una comprensión, no solo del disciplinamiento de las individualidades, sino que también del "desapego" (Araujo, 2019: 33) producido por la combinatoria de años de dictadura y neoliberalismo, entendiendo por desapego la distancia — a veces desconfiada, otras resignada o impotente - respecto de las normas y los valores que regulan la vida en común. Modo imperativo y uso de la exclamación enfatizan el carácter directivo 
del escrito, le otorgan buena parte de su "fuerza ilocucionaria" (Searle 1982, 2001) pero, además, buscan producir el impulso necesario para generar un movimiento anti-sistema entre individuos desapegados para que actúen ahora. En este último sentido, el modo imperativo y el uso de la exclamación parecen sugerir la necesidad de acometer una acción en presente, ahora mismo, en la urgencia de un escenario asediado por las exhortaciones que hemos indicado anteriormente y cuyo enfrentamiento requiere de una acción inmediata. Se esboza aquí una pista de reflexión interesante en cuanto al horizonte temporal de la acción solicitada por el escrito o, mejor aún, sobre el "régimen de historicidad" (Hartog, 2003) convocado por el escrito, toda vez que la noción de "régimen de historicidad" alude a la modalidad de autoconciencia de una comunidad humana. En otras palabras, pareciera que el escrito "EVADE!" remece el modo en que hemos compaginado y en que vivenciamos pasado, presente y futuro.

Sabemos, por lo que hemos dicho en las páginas precedentes, que la fuerza de un escrito expuesto en la ciudad no depende solo del enunciado, sino que también de su despliegue y su exposición. Por ello, un cuarto aspecto que amerita nuestra atención remite a lo que anteriormente denominamos "propagación" del escrito.

A este respecto debemos retener el hecho de que "EVADE!" es una intervención sobre la materialidad del espacio público y que este tipo de intervenciones no son nunca triviales. Al contrario, usualmente son vistas como formas de apropiación y de puesta en cuestión de la normatividad del espacio y no solo como formas de expresión personal o colectiva.

En ese sentido, conviene pensar en la ciudad no solo como una materialidad provista de significado, sino que como un producto de la actividad humana que permanentemente está actuando sobre nuestra percepción y nuestra sensibilidad, orientándola y definiéndola: "Cada ciudad obliga a vivir y a ser de ciertas maneras" (Remedi, 2006: 105).

Por ello, cuando intervenimos la ciudad, estamos haciendo visibles las delimitaciones organizativas y sensibles de nuestro mundo. Y, más aún, estamos sugiriendo, a los receptores de nuestra intervención, que modificar 
estas delimitaciones es posible. De hecho, el escrito en el espacio público persuade, antes que de su contenido mismo, de la posibilidad y validez de intervenir ese espacio. En consecuencia, cuando la presencia de estas intervenciones gráficas se incrementa, aumenta también la posibilidad de que ellas proliferen, lo que redunda en que se acreciente su potencial persuasivo. Con referencia al grafiti político Fraenkel (2007) ofrece una perspectiva que también resulta válida para el escrito que aquí analizamos: el grafiti pasa a estar "integrado en el entorno (...) dura (...) se impone cotidianamente a la vista del transeúnte" y es justamente la "permanencia de la inscripción [lo que] sugiere al militante que el enunciado se puede realizar" (Fraenkel, 2007: 104). ${ }^{4}$ Un razonamiento análogo podríamos proponer en relación a la reiteración de un escrito en la ciudad o a su radio de cobertura en el espacio urbano: la presencia material del escrito se convierte en una fuente de incitación y persuasión para sus destinatarios. Pero, ¿persuasión respecto de qué?

Efectivamente, en "EVADE!" no hay un desarrollo argumental o proposicional acabado. No hay un despliegue narrativo ni una política afirmativa. Tampoco hay una propuesta alternativa de modelo de desarrollo. Sin embargo, se trata de un poderoso detonador de nuestro trasfondo interpretativo y una pieza que actualiza nuestro repertorio de acción. Ahí radica su potencia política.

"Escribir grafitis políticos forma parte del 'repertorio de acción colectiva", plantea Fraenkel aludiendo a la obra de Charles Tilly, a pesar de que este último no se refiera a los actos de escritura como un recurso específico, ni de la misma relevancia que las huelgas, las ocupaciones de tierras o las apropiaciones de alimentos. En defensa de su argumento, Fraenkel indica que los grafitis responden a normas escriturales "que no son explícitas ni están instituidas, pero que no están por ello menos reguladas por una memoria activista y de prácticas de imitación de modelos conocidos" (Fraenkel, 2017: 102). La noción de repertorio de acción colectiva es bastante amplia. Para

4 Traducción del autor. 
Tilly (2002), se trata de un conjunto de prácticas aprendidas a través de la experiencia de protesta, moldeado por las formas de organización de los grupos, sus recursos y su experiencia, entre otros factores. Los repertorios "surgen de la lucha" ya que es "en la protesta donde la gente aprende" (Tilly, 2002: 31), vale decir, el repertorio se actualiza a través de las ejecuciones y siempre se encuentra "en proceso", rehaciéndose continuamente. Otro autor de referencia en el análisis de los movimientos sociales, Sidney Tarrow, ha indicado que los nuevos repertorios de acción colectiva tienden a un menor uso de la violencia en sus formas de confrontación en comparación a los repertorios empleados en tiempos pasados (Tarrow, 1997), sin que por ello pierdan sus características fundamentales, como son la búsqueda de la mejor oportunidad y de la mayor eficacia posible, incluso a través del uso de estrategias menos directas y más dispersas (Tilly, 2007).

El escrito "EVADE!" responde a estas características: puede ser pensado como una práctica gráfica que forma parte del repertorio de acción de grupos militantes o activistas, que con su intervención del espacio público, actualiza ese repertorio, pero, además, en función de su proliferación, reiteración y persistencia, ese escrito interviene sobre el repertorio de lo pensable de todos sus destinatarios y receptores, integrando un componente poco usual: la evasión. Evadir se convierte, así, en una acción posible de realizar para hacer frente a un alza de precio considerada injusta, frente a las conminaciones a la adaptación y a la responsabilización individual, frente al pretendido control de nuestras aspiraciones y deseos, frente al disciplinamiento de nuestros comportamientos.

Hay aquí una dimensión política importante del escrito "EVADE!". No se trata del llamado a la movilización de un colectivo, ni la propuesta de un enfrentamiento o una lucha contra el sistema. Tampoco hay constatación de una contradicción estructural. Más bien hay un reconocimiento tácito de la imposibilidad de transformar completa e inmediatamente la sociedad o de proponer un modelo alternativo, solo que, al mismo tiempo, se sugiere que el actual no es el único orden posible, aceptable o deseable. En este sentido, "EVADE!" es, posiblemente, uno de los mejores ejemplos de escrito 
"heterotópico" (Foucault, 1984), pues no señala un lugar de destino en el que no existe la opresión y reina el bienestar colectivo, sino que, más modestamente, alude a una posiblidad lateral, una alternativa que está aquí, ahora, al alcance de cualquiera que se decida, a actuar y a habitar la alternativa (Kanngieser 2015).

De esta manera, este escrito sencillo, simple y directo consigue darle forma a algo que antes no era más que ruido, alterando la habitual distribución de las legitimidades para hablar y las competencias para ver (Rancière 2000, 2004). Propagado en el espacio público se instala como una evidencia sensible que pone al descubierto una de las aristas de nuestra vida en común y parte de las regulaciones que determinan nuestra participación y comportamiento frente a ese común (Rancière, 2000). Se convierte en escrito político.

\section{CONCLUSIÓN}

Frente a la potencia de los medios de comunicación y la ubicuidad de las redes sociales; frente a la saturación publicitaria y comercial de nuestras calles, ¿qué rol puede jugar el escrito en el espacio público? Esta interrogante, en cierta forma sustraída y parafraseada de la reflexión hecha por Valérie Tesnière (2012: 8), resulta crucial para situar nuestra propia reflexión sobre "EVADE!".

La discusión sobre los escritos en el espacio público suele estar atravesada por los lugares comunes del vandalismo, la suciedad y el daño, así como circunscrita a una versión del patrimonio y de la estética fuertemente despolitizados. Sin embargo, ese mismo encuadre discursivo da cuenta de la importancia asignada al control visual y escritural del espacio público (Artières, 2013; Araya, 2010) y revela la condición estratégica de este último en tanto interfaz política entre el poder y los ciudadanos. Esos discursos pocas veces reconocen un asunto que para nostoros es fundamental: que las intervenciones gráficas activan la condición pública del espacio y la sostienen en el tiempo a través de su actuar irritante.

A pesar de su condición casi microscópica, no debiéramos olvidar 
que los escritos callejeros son indisociables del debate político debido a su materialidad misma, a su inevitable condición expuesta en el espacio público y su consiguiente intervención sobre nuestros registros sensibles. Pero también porque son un recurso al alcance de la mano, aún no coopatado por los poderes disciplinarios: se escribe en la calle para actuar y para traer de vuelta la política a la ciudadanía. En este sentido, el escrito "EVADE!" ratifica que la escritura en el espacio público urbano es todavía una forma posible de transformar el equilibrio precario de nuestros disciplinamientos y una práctica apropiada para modificar las normas que regulan, no solo la escritura, sino que nuestras acciones y comportamientos.

Debido a su condición microscópica, los escritos callejeros a veces proliferan y se vuelven epidémicos, actuando políticamente sobre vastas poblaciones e interviniendo, como dice Rancière (2000), sobre las propiedades de los espacios y los posibles del tiempo.

Parafraseando una vez más a Fraenkel (2018), podríamos decir que nuestras subjetividades, luego del estallido social de octubre de 2019, se construirán también, en parte, a partir de las inscripciones gráficas de la calle, convertidas esta vez en recursos memoriales, congnitivos e imaginarios para componer un nuevo lazo y dar forma a otra política.

\section{REFERENCIAS}

Austin, J. (2003). Cómo hacer cosas con palabras. Editorial Paidós, Buenos Aires.

Alarcón S, M. (2008). Austin y Searle: la relación entre verbos y actos ilocucionarios. Literatura y lingüística, (19), 235-250. https://dx.doi. org/10.4067/S0716-58112008000100013

Araujo, K. (2019). “Desmesuras, desencantos, irritaciones y desapegos”. En Araujo, K., Hilos tensados. Editorial USACH, Santiago. 
Araya, P. (2007), "Marcas expuestas, poéticas contestatarias. Escrituras en el espacio urbano chileno", en Teresa Orecchia-Havas (dir.), Les villes et la fin du XX eme. siècle en Amérique Latine: Littératures, cultures, représentations, Bern, Suiza: Peter Lang, 435-454.

(2008), "El Mercurio Miente: Siete notas sobre escrituras expuestas", Revista Austral de Ciencias Sociales, 14: 157-172.

(2010), "NO + (Chile 1983-2007). Uwagi o pismie kontestacyjnym. W strone pragmatycznej antropologii pisma", en Ph. Artières y P. Rodak, Antropologia pisma. Od teorii do praktyki, (Antropologia de la escritura. De la teoría a la práctica), Varsovia, Polonia: WUW, 93-113. Artières, P. (2013). La police de l'écriture. L'invention de la délinquance graphique 1852-1945. Ediciones La Découverte, París.

Barthes, R. (1984) L'écriture de l'événement. En Barthes, R. Le Bruissement de la langue, 175-181. Ediciones Seuil, París.

Benveniste, E. (1976). La philosophie analytique et le langage. En Benveniste, E. Problèmes de linguistique générale, tomo I, 275-276.

Bosredon, B. (1997), Les titres de tableaux. Une pragmatique de l'identification. PUF, París.

Campos, L. (2009). Los murales de La Victoria: efectos de sentido y lugar. Actuel Marx/Intervenciones, 8, 129-142.

(2014). "Espero tu ayuda" o el proceso de reconstrucción de Valparaíso desde la perspectiva de un habitante. Revista Territorio $F A U, 1,21-25$.

(2019). "Graphic Inscriptions and the Production of Urban Public Space in Valparaiso, Chile”, en Hernández-García, Cárdenas-O’Byrne, S., García-Jerez, A., Beza, B. (Editores) "Urban Space: Experiences and Reflections from the Global South". Sello Editorial Javeriano.

Campos, L. y Aguilar, M. A. (2015). “L'expérience corporelle de l'attente dans les déplacements en métro". En Vidal, L. y Musset, A. (coord.) Les territoires de l'attente. Migrations et mobilités dans les Amériques (XIX - XXI siècle), Presses Universitaires de Rennes. 
Campos, L. y Suazo, V. (2019). “Escribir las relaciones y el territorio”. ÍCONOS 64: 223-231 DOI: http://dx.doi.org/10.17141/iconos.63.2019.3394

Denis, J. y Pontille, D. (2009). L'écologie informationnelle des lieux publics: Le cas de la signalétique du métro. En Licoppe, C. L'évolution des cultures numériques, de la mutation du lien social à l'organisation du travail. Ediciones FYP, 94-101.

(2013). Ficelles pour une ethnographie de l'écrit. En Datchary, C. Petit précis de méthodologie. Le sens du détail dans les sciences sociales. Ediciones Le bord de l'eau, 17-30.

Foucault, M. (1984). «Des espaces autres» Conférence au Cercle d'études architecturales, 14 mars 1967. En Dits et Ecrits, 1571-1581. Ediciones Gallimard, París.

(2004). La Naissance de la biopolitique. Cours au Collège de France (1978-1979). Ediciones Le Seuil, París.

Fraenkel, B. (2006). Actes écrits, actes oraux : la performativité à l'épreuve de l'écriture. Études de communication, 29, 69-93. Retrieved from http:// edc.revues.org/369.

(2007). Actes d'écriture: quand écrire c'est faire. Langage et Société, 3, 101- 112. DOI : $10.3917 / \mathrm{ls} .121 .0101$

(2008). Las escrituras de la catástrofe. Práctica de escritura y de lectura en la ciudad de Nueva York en septiembre 2001. Actuel Marx / Intervenciones, 6, 157-172.

(2017). Actos de escritura: cuando escribir es hacer. Thémata. Revista de Filosofia $\mathrm{N}^{\circ}$ 56, julio-diciembre (2017) : 319-329. ISSN: 0212-8365 e-ISSN: 2253-900X

(2018). La notion d'événement d'écriture. Communication \& langages 2018/3 $\mathrm{N}^{\circ} 197,35-52$.

Garretón, M. A. (2012). Neoliberalismo corregido y progresismo limitado: los gobiernos de la Concertación en Chile 1990-2010. Santiago: Arcis/ CLACSO.

Hartog, F. (2003). Régimes d'historicité. Présentisme et expérience du temps. Ediciones Le Seuil, París. 
Kanngieser, a. (2012). ... And ... and ... and ... The Transversal Politics of Performative Encounters. En Deleuze Studies 2012 6: 2, 265-290

Latour, B. \& Hermant, E. (1998). Paris ville invisible. Paris : Les empêcheurs de penser en rond/La Découverte.

Latour, B. \& Weibel, P. (dir.) 2005. Making things public: Atmospheres of democracy. Cambridge, MIT Press.

Miller, D. (Editor) (1998). Material Cultures. Why some things matter. UCL Press. London. (2005). Materiality. Duke University Press. EE.UU.

Pellegram, A. (1998). The message in paper. En Miller, D. Material Cultures. Why some things matter. UCL Press. London. 103-120.

Petrucci, A. (1993). Jeux de lettres. Formes et usages de l'inscription en Italie 11e-20e siècles. Paris: Éditions de l'EHESS.

Rancière, J. (2000). Le partage du sensible. Esthétique et politique. Ediciones La Fabrique, París. (2004). Malaise dans l'esthétique. Ediciones Galilée, París.

Remedi, G. (2006). "Ciudad letrada: Ángel Rama y la espacialización del análisis cultural”, en Moraña, M. (Editor), Ángel Rama y los estudios latinoamericanos Introducción, Ediciones Universidad de Illinois, 97122.

Shusterman, R. (2015). Le Style à l'état vif: somaesthétique, art populaire et art de vivre. Ediciones Questions théoriques, París.

Searle, J. (1982). Sens et expression. Les Editions de Minuit, París. (1997). La construcción de la realidad social. Editorial Paidós, Barcelona.

(2001). Actos de habla. Ensayo de filosofía del lenguaje. Editorial Cátedra, Madrid.

Stiegler, B. (2019). "Il faut s'adapter". Sur un nouvel impératif politique. Ediciones Gallimard, París.

Tarrow, S. (1997). El poder en movimiento. Los movimientos sociales, la acción colectiva y la política. Madrid, Alianza. 
Tesnière, V. (2012). "Affiche-Action: Quand la politique s'écrit dans la rue". En Fraenkel, B., Gouiran, M., Jakobowicz, N. y Tesnière, V. AfficheAction: Quand la politique s'écrit dans la rue. Ediciones Gallimard/ BDIC, París.

Tilly, Ch. (2002). "Repertorios de acción contestataria en Gran Bretaña: 17581834”, en: Traugott, M. Protesta social. Editorial Hacer, Barcelona. (2007). Violencia colectiva. Editorial Hacer, Barcelona. 